\title{
Plant PKS structure revealed
}

To date, very little structural information has been garnered on polyketide synthases (PKSs), enzymes responsible for producing a wide variety of metabolites, from plant pigments/antimicrobial phytoalexins to streptomycete macrolide antibiotics. Now, an international team has determined for the first time the structure of a simple plant PKS, chalcone synthase (CHS), to $1.56 \AA$ resolution (Nat. Struct. Biol. 6, 775-784, 1999). By expressing wild-type and mutant forms of alfalfa CHS in Escherichia coli and crystallizing the purified enzyme, they were able to determine the structure in complex with substrate and product analogs using $\mathrm{x}$-ray crystallography. "The most surprising aspect of the crystal structure was the simplicity of the active site," says Joseph Noel, the corresponding author on the paper. Homodimeric CHS produces chalcone through a complex set of reactions, including sequential condensation, carbon-carbon bond ligations, ring closure, and aromatization. But the structure reveals remarkably few chemically reactive residues within the two functionally independent active sites. The work also has implications for other PKSs: "About one quarter of the related enzymes with sequences 60-80\% identical to CHS have different active-site volumes, which suggests that these enzymes carry out completely novel reactions," says Noel. What's more, the CHS structure should be useful in elucidating reaction mechanisms in more complicated multidomain macrolide PKSs.

\section{Ex vivo stem cell culture}

Scientists have succeeded in maintaining hematopoietic stem cells (HSCs) outside the body in a pluripotent state for the first time. By culturing explanted murine HSCs in the presence of growth factor thrombopoietin (TPO), researchers at the University of Washington (Seattle, WA) have established long-term bone marrow cultures in which HSCs can reproduce for at least four months. The findings, reported in PNAS $(14,8126-8131,1999)$ could pave the way to a variety of new clinical applications. The complexity of bone marrow has made it difficult for researchers to duplicate ex vivo the conditions encountered by stem cells in vivo. As a result, explanted HSCs lose their pluripotency and differentiate into particular blood cell lineages after a short time in culture. Corresponding author Stephen Bartelmez says the team found that of the three major cell types involved in the replication of the stem cell-marrow fibroblasts, marrow endothelial cells, and megakaryocytes-megakaryocytes were absent. "That's why it took so long to crack the nut," he says. But when cells were cultured with TPO for several weeks, some of the HSCs did differentiate into megakaryocytes, providing the missing component required for longterm HSC survival.

Research News Briefs written by Aseem Ansari, Alan Dove, and Andrew Marshall.

\section{Ribozyme, snorbozyme}

By fusing a hammerhead ribozyme to a small nucleolar RNA, researchers have created an artificial ribozyme, dubbed a "snorbozyme," which localizes to the nucleolus of a yeast cell and cleaves a target RNA with nearly perfect efficiency. The new work (PNAS 96, 6609-6614, 1999) may provide an approach to gene control for both basic research and clinical use. Hammerhead ribozymes can be engineered to cleave almost any RNA molecule, but the relatively low efficiency of artificial ribozymes in vivo has limited their use. Reasoning that colocalization of a ribozyme and its RNA substrate in the same cellular compartment would result in more efficient cleavage, the scientists used small nucleolar RNAs (snoRNAs) as both catalysts and targets. The snorbozyme is metabolically stable, and is directed to the nucleolus with high specificity. Kinetic analysis showed that the snorbozyme cleaves its target snoRNA with an efficiency approaching 100\%, functionally knocking out the snoRNA gene. Maurille Fournier, a senior author on the paper, says that as many as 200 different snoRNAs could be targeted with this approach, and it may also be possible to modify the technique to cleave specific mRNA molecules in the nucleoplasm. "As a research tool, there are just lots of wonderful opportunities for selectively knocking out genes and gene products in a natural cellular environment," says Fournier.

\section{Anticancer verotoxin}

An Escherichia coli toxin responsible for food-poisoning outbreaks associated with contaminated hamburgers might have a more palatable role as an anticancer agent. In a paper in Oncology Research $(11,33-39$, 1999), scientists at the Hospital for Sick Children (Toronto, Ontario) and the University of Toronto report using the verotoxin 1 protein to treat human astrocytomas xenografted into nude mice. Tumors injected with the toxin regressed completely and did not return, and the treated mice suffered no obvious side effects. Senior author Clifford Lingwood says that no other treatment has shown similar efficacy in the mouse system. In "hamburger disease", verotoxin targets a receptor, found in the developing blood vessels, that is absent from the glomeruli of adult kidneys, but is expressed in newly developing blood vessels of astrocytomas, as well as some multidrug-resistant tumors. In the mouse system, verotoxin caused the grafted astrocytomas and their associated blood vessels to undergo apoptosis. "As tumor cells become resistant to standard chemotherapy, they become more sensitive to this approach," Lingwood adds. Ottawabased company Select Therapeutics plans to develop verotoxin for clinical use, and phase I trials are currently being designed.

\section{Abzyme prodrugs}

An elegant combination of catalytic antibody and a chemical cloaking approach has been used to target two common anticancer drugs specifically to tumor cells. To achieve this, Carlos Barbas and colleagues first masked the reactive functional groups of two known anticancer drugs, doxorubicin and camptothecin, to make them generally less toxic to healthy cells (Proc. Natl. Acad. Sci USA 96, 6925-6930, 1999). Cunningly, the chemical they used to cloak the drugs could not be removed by physiological enzymes, but was readily uncoupled by a catalytic antibody (38C2) that they had previously isolated. Experiments in which they tested the effectiveness of activating the prodrugs in cell culture on colon and prostrate cancer cells showed the antibody to be effective at therapeutically relevant concentrations. In addition, when injected intravenously into mice, the catalytic antibody remained catalytically active over several weeks. Since the chemical cloaking method is generally applicable to many drugs, Barbas suggests the approach would be valuable for targeting aberrant cells with any prodrug of choice, once the antibodies have been adapted for human use. 